\section{Evaluation of Lettuce Genotypes for Seed Thermotolerance}

\author{
Abbas Lafta and Beiquan Mou' \\ U.S. Department of Agriculture (USDA), Agricultural Research Service, \\ 1636 East Alisal Street, Salinas, CA 93905
}

Additional index words. Lactuca sativa, thermoinhibition, thermodormancy, crisphead lettuce, butterhead lettuce, romaine lettuce

\begin{abstract}
Thermoinhibition of lettuce (Lactuca sativa L.) seed germination is a common problem associated with lettuce production. Depending on lettuce cultivars, seed germination may be inhibited when temperatures exceed $28^{\circ} \mathrm{C}$. The delay or inhibition of seed germination at high temperatures may reduce seedling emergence and stand establishment of lettuce in the field, leading to a reduction in economic yield. To identify heat-tolerant lettuce genotypes, lettuce varieties and germplasm accessions were screened for the ability to germinate under high-temperature stress. Twenty-four to 26 genotypes were selected from each lettuce types (crisphead, romaine, butterhead, loose leaf, and wild species) and their seeds were placed in petri dishes to test their ability to germinate at high temperatures $\left(29\right.$ and $34{ }^{\circ} \mathrm{C}$ ) as compared with controls at $24^{\circ} \mathrm{C}$. Some lettuce genotypes showed thermotolerance to $34^{\circ} \mathrm{C}$ (less than $20 \%$ reduction in germination) such as Elizabeth, PI 342533, PI 358025, Florida Buttercrisp, Kordaat, Corsair, FL 50105, PRO 425, PI 278070, Noemie, Picarde, Gaillarde, L. serriola (PI 491112, UC96US23, PI 491147), L. virosa (PI 274378 D), L. saligna (PI 491159), and primitive (PI 187238 A, PI 289063 C). The germination rates were consistent with the germination percentage at the high temperatures. Seed germination in the field was very low and positively correlated with seed germination at 29 and $34^{\circ} \mathrm{C}$. The highest field germination percentages (greater than $40 \%$ ) were observed in Belluro, Mantilia, Mid Queen, Headmaster, PRO 874, PRO 425, FL 50105, Corsair, Romaine SSC 1148, Romaine Romea, Green Forest, Grenadier, FL 43007, Squadron, Xena, Noemie, Green Wave, Picarde, and Red Giant. The results of this study indicated that lettuce genotypes differ greatly in their ability to germinate at high temperatures as determined by the percentages and the rates of germination. Our research indicates that thermoinsensitive varieties could be used to expand lettuce production seasons in warm and low land cost areas and reduce the need for seed priming, lowering the production costs. The information may also be useful for growers to better choose cultivars for warm environments and for lettuce breeders to improve the crop for adaptation to global warming and climate change.
\end{abstract}

Temperature is a major environmental factor that influences plant growth and development. Lettuce (Lactuca sativa L.) is a cool-season crop with optimum growth at an average temperature of $18{ }^{\circ} \mathrm{C}$. Production of lettuce at higher temperature ranges results in yield and quality losses (Jenni, 2005; Jenni and Yan, 2009). At high temperatures, lettuce seed germination is inhibited (thermoinhibition) and the seeds became dormant, which is called thermodormancy (Gonai et al., 2004; Negm et al., 1972; Vidaver and Hsiao, 1975). It has been reported that seed germination at high temperatures is influenced by the environmental conditions, especially high temperatures, during seed development and maturation (Kozarewa et al., 2006; Sung et al., 1998, 2008). Poor seed germination and thermodormancy are major problems associated with lettuce production. Depending

Received for publication 13 Feb. 2013. Accepted for publication 25 Apr. 2013.

The USDA is an equal opportunity provider and employer.

${ }^{1}$ To whom reprint requests should be addressed; e-mail beiquan.mou@ars.usda.gov.
(Ikuma and Thimann, 1963; Speer, 1974; Sung et al., 1998). Weakening of the endosperm layer before radicle emergence through enzymatic activity of endo- $\beta$-mannanase, a cell wall-bound enzyme, is essential for seed germination to occur at high temperatures (Nascimento et al., 2000). This suggested that seed priming overcomes the thermoinhibition in thermosensitive lettuce cultivars as a result of an increase in enzyme activity in the endosperm layer of the seed. The endosperm layer is considered the region that imposes physical resistance to seed germination at high temperature (Sung et al., 2008). The balance between embryo growth potential and the physical resistance to embryo growth exerted by the covering tissues is required for dormancy release and seed germination to occur (Kucera et al., 2005).

The plant hormones, gibberellic acid (GA), abscisic acid (ABA), and ethylene play a role in the regulation of seed germination in most plants. Seed germination is inhibited by $\mathrm{ABA}$, which increases in thermodormant lettuce seeds (Argyris et al., 2008a). GA is involved in dormancy release or prevention by stimulating the activities of hydrolytic enzymes, which promote embryo growth (Khan, 1994). Ethylene may also play a role in extending the high temperature limit for lettuce seed germination by maintaining lower water potential in the embryonic region to allow growth and radicle emergence (Dutta and Bradford, 1994). Nascimento et al. (2000) observed a close relationship among lettuce seed germination, ethylene evolution, and the activity of endo- $\beta$-mannanase enzyme. Ethylene and cytokinin have been reported to be involved in alleviation of thermoinhibition in lettuce seeds (Huang and Khan, 1992; Khan and Prusinski, 1989).

The average temperature worldwide is predicted to increase over time, which impacts the agricultural production and food supplies (Karl and Trenberth, 2003; Wurr et al., 1996). This climate change and global warming may pose serious challenges to California agriculture, especially the leafy green industry. Adapting the leafy green industry to future climate conditions is important to meet the increasing demand for leafy vegetables as the population increases. The increasing need for leafy vegetables will put pressure on the leafy industry to expand the production to the low land cost and warmer areas of California. To expand lettuce production to warmer environments, lettuce germplasm and cultivars need to be evaluated to examine their tolerance to high-temperature stress. Lettuce seed germination is inhibited at high temperatures, which leads to a reduction in product quality and yield. Thus, thermoinhibition is a problem facing lettuce growers, which could be solved by selecting thermotolerant varieties that perform well under stressful conditions. The objective of this study was to screen lettuce germplasm and cultivars for resistance to thermoinhibition or thermodormancy to find lettuce germplasm that germinate well at high temperatures. We screened the lettuce collections at the U.S. Department of Agriculture (USDA) in 
Salinas, CA, for their tolerance to thermoinhibition or thermodormancy and identified the most thermotolerant genotypes from different lettuce types such as crisphead, butterhead, romaine, leaf type, and wild species.

\section{Materials and Methods}

Plant material. Five lettuce types were screened in this experiment to test their tolerance to thermoinhibition including crisphead, butterhead, romaine, loose leaf (green and red leaf), and wild species. Initially, we screened more than 3500 lettuce varieties and germplasm accessions for their ability to germinate at high temperature. Twenty-five to 26 genotypes from each lettuce type were selected to be used in further experiments, including genotypes with a high germination percentage at $34{ }^{\circ} \mathrm{C}$ and some standard and thermosensitive genotypes. The selected genotypes have uniform seed germination at $24^{\circ} \mathrm{C}$ and their seeds were produced in greenhouses at the USDA, Salinas, CA, and stored at $-20{ }^{\circ} \mathrm{C}$. This may minimize the effect of the environmental conditions, at which the seeds were matured, on seed germination.

Seed germination. Four replicates of 25 seeds each were placed in petri dishes $(100 \times 20 \mathrm{~mm})$ over one layer of Whatman \#1 filter paper and $4.5 \mathrm{~mL}$ of deionized water was added. The petri dishes were covered with lids to prevent evaporation. The petri dishes were placed in incubators maintained at 24,29 , and $34^{\circ} \mathrm{C}$ under a 12 -h fluorescence light $\left(80 \mu \mathrm{mol} \cdot \mathrm{m}^{-2} \cdot \mathrm{s}^{-1}\right)$ for $14 \mathrm{~d}$. We used this light intensity because the optimal temperature for seed germination in lettuce was noted to be higher in light than in darkness (Deng and Song, 2012). Seed germination was recorded as the emergence of the radicle after 2, 4,7 , and $14 \mathrm{~d}$ or until no additional germination occurred. Percentage of seed germination was calculated and the germination rate was determined based on the method of Meguire (1962) using the following equation: germination rate $=\sum \mathrm{GT} 1 / \mathrm{T} 1+\longrightarrow+\mathrm{GTn} / \mathrm{Tn}$. GT1 $=$ number of germinated seeds on first count; $\mathrm{GTn}=$ number of germinated seeds on last count; $\mathrm{T} 1=$ days at first count; $\mathrm{Tn}=$ days at last count. The percentage reduction in seed germination at 29 and $34{ }^{\circ} \mathrm{C}$ from that at $24{ }^{\circ} \mathrm{C}$ was also calculated.

Field germination. Seeds of lettuce genotypes were planted on 10 July 2012 in a field at the West Side Research and Extension Center, University of California, Five Points, $\mathrm{CA}$. The experiment was arranged in a randomized complete block with four replications per treatment. Fifty seeds from each genotype were planted in rows $6 \mathrm{~m}$ long. The experimental unit consisted of one row per entry. Seed germination was evaluated after 7 and $14 \mathrm{~d}$. The average maximum and minimum air temperatures for the $14 \mathrm{~d}$ were 34.9 and $16.1{ }^{\circ} \mathrm{C}$ and the average maximum and minimum soil temperatures at $15-\mathrm{cm}$ depth were 27.2 and $24.9^{\circ} \mathrm{C}$, respectively.

Statistical analysis. Analysis of variance was conducted using the JMP program (SAS Institute Inc., Cary, NC). Treatment means were separated by the least significant difference at the 0.05 level of probability. The correlation coefficients between field seed germination and germination at 29 and $34{ }^{\circ} \mathrm{C}$ were determined by the JMP program using genotype means.

\section{Results and Discussion}

Butterhead lettuce. All the butterhead genotypes showed a high germination percentage and germination rate at $24^{\circ} \mathrm{C}$ (Table 1). Annecy exhibited the lowest germination percentage (83\%) and germination rate among all genotypes tested. There were highly significant differences in germination percentage and germination rates among genotypes at 29 and $34{ }^{\circ} \mathrm{C}$. At $29{ }^{\circ} \mathrm{C}$, Annecy, Anthem, Dark Green Boston, and Winter Marvel showed the lowest germination percentage and germination rates compared with other genotypes. The reductions in seed germination at $29{ }^{\circ} \mathrm{C}$ in these were $54 \%, 93 \%, 70 \%$, and $100 \%$, respectively (Fig. 1). These genotypes were the most sensitive genotypes to thermoinhibition at $29^{\circ} \mathrm{C}$. Significant cultivar

Table 1. Effect of temperature on seed germination in butterhead lettuce.

\begin{tabular}{|c|c|c|c|c|c|c|c|}
\hline \multirow[b]{2}{*}{ Genotype } & \multicolumn{3}{|c|}{ Germination (\%) } & \multicolumn{3}{|c|}{ Germination rate } & \multirow{2}{*}{$\begin{array}{c}\text { Field } \\
\text { germination }(\%)\end{array}$} \\
\hline & $24{ }^{\circ} \mathrm{C}$ & $29^{\circ} \mathrm{C}$ & $34{ }^{\circ} \mathrm{C}$ & $24{ }^{\circ} \mathrm{C}$ & $29^{\circ} \mathrm{C}$ & $34^{\circ} \mathrm{C}$ & \\
\hline Annecy & $83 b^{z}$ & $37 \mathrm{c}$ & $3 \mathrm{~h}$ & $34.0 \mathrm{~d}$ & $10.1 \mathrm{~cd}$ & $0.4 \mathrm{i}$ & $2 \mathrm{i}$ \\
\hline Anthem & $100 \mathrm{a}$ & $7 \mathrm{~d}$ & $4 \mathrm{~h}$ & $50.0 \mathrm{a}$ & $2.5 \mathrm{de}$ & 0.91 & $1 \mathrm{i}$ \\
\hline Aquarius & $100 \mathrm{a}$ & $78 \mathrm{~b}$ & $11 \mathrm{gh}$ & $48.0 \mathrm{ab}$ & $28.6 \mathrm{~b}$ & $2.3 \mathrm{i}$ & $40 \mathrm{abc}$ \\
\hline Arcade & $100 \mathrm{a}$ & $100 \mathrm{a}$ & $11 \mathrm{gh}$ & $50.0 \mathrm{a}$ & $47.5 \mathrm{a}$ & $3.4 \mathrm{i}$ & $33 \mathrm{a}-\mathrm{e}$ \\
\hline Averya & $97 \mathrm{a}$ & $93 \mathrm{a}$ & $69 \mathrm{~cd}$ & $45.7 \mathrm{~b}$ & $44.3 \mathrm{a}$ & $22.0 \mathrm{def}$ & $14 \mathrm{ghi}$ \\
\hline Belluro & $100 \mathrm{a}$ & $100 \mathrm{a}$ & $65 \mathrm{~d}$ & $50.0 \mathrm{a}$ & $50.0 \mathrm{a}$ & $18.3 \mathrm{fg}$ & $44 \mathrm{a}$ \\
\hline Bibb & $100 \mathrm{a}$ & $100 \mathrm{a}$ & 39 ef & $50.0 \mathrm{a}$ & $50.0 \mathrm{a}$ & $19.5 \mathrm{efg}$ & 8 hi \\
\hline Big Hoss & $100 \mathrm{a}$ & $97 \mathrm{a}$ & $65 \mathrm{~d}$ & $49.3 \mathrm{a}$ & $48.5 \mathrm{a}$ & $30.9 \mathrm{c}$ & $19 \mathrm{fgh}$ \\
\hline Buttercrunch & $100 \mathrm{a}$ & $94 \mathrm{a}$ & 38 ef & $50.0 \mathrm{a}$ & $46.6 \mathrm{a}$ & $19.0 \mathrm{fg}$ & $28 \mathrm{~b}-\mathrm{g}$ \\
\hline Calibra & $100 \mathrm{a}$ & $100 \mathrm{a}$ & $55 \mathrm{de}$ & $49.0 \mathrm{a}$ & $46.8 \mathrm{a}$ & $7.6 \mathrm{hi}$ & 9 hi \\
\hline Clinton & $100 \mathrm{a}$ & $100 \mathrm{a}$ & $73 \mathrm{bcd}$ & $50.0 \mathrm{a}$ & $50.0 \mathrm{a}$ & $20.2 \mathrm{ef}$ & $20 \mathrm{e}-\mathrm{h}$ \\
\hline Dark Green Boston & $96 \mathrm{a}$ & $29 \mathrm{c}$ & $3 \mathrm{~h}$ & $40.3 \mathrm{c}$ & $13.6 \mathrm{c}$ & $1.3 \mathrm{i}$ & 9 hi \\
\hline Deciso & $100 \mathrm{a}$ & $100 \mathrm{a}$ & $26 \mathrm{fg}$ & $50.0 \mathrm{a}$ & $50.0 \mathrm{a}$ & $12.3 \mathrm{gh}$ & $1 \mathrm{i}$ \\
\hline Dukaat & $100 \mathrm{a}$ & $100 \mathrm{a}$ & $56 \mathrm{de}$ & $50.0 \mathrm{a}$ & $50.0 \mathrm{a}$ & 26.8 cde & $25 \mathrm{~d}-\mathrm{g}$ \\
\hline Elizabeth & $100 \mathrm{a}$ & $100 \mathrm{a}$ & $98 \mathrm{a}$ & $50.0 \mathrm{a}$ & $50.0 \mathrm{a}$ & $46.7 \mathrm{ab}$ & $14 \mathrm{ghi}$ \\
\hline Florida Buttercrisp & $100 \mathrm{a}$ & $100 \mathrm{a}$ & $88 \mathrm{abc}$ & $50.0 \mathrm{a}$ & $50.0 \mathrm{a}$ & $41.4 \mathrm{ab}$ & $29 \mathrm{~b}-\mathrm{f}$ \\
\hline Kitty & $100 \mathrm{a}$ & $100 \mathrm{a}$ & $58 \mathrm{de}$ & $50.0 \mathrm{a}$ & $50.0 \mathrm{a}$ & $28.6 \mathrm{~cd}$ & $32 \mathrm{a}-\mathrm{f}$ \\
\hline Kordaat & $100 \mathrm{a}$ & $100 \mathrm{a}$ & $88 \mathrm{abc}$ & $50.0 \mathrm{a}$ & $50.0 \mathrm{a}$ & $41.0 \mathrm{~b}$ & $37 \mathrm{a}-\mathrm{d}$ \\
\hline Magnet & $100 \mathrm{a}$ & $100 \mathrm{a}$ & $69 \mathrm{~cd}$ & $50.0 \mathrm{a}$ & $50.0 \mathrm{a}$ & $31.5 \mathrm{c}$ & $37 \mathrm{a}-\mathrm{d}$ \\
\hline Mantilia & $100 \mathrm{a}$ & $100 \mathrm{a}$ & $62 \mathrm{~d}$ & $50.0 \mathrm{a}$ & $50.0 \mathrm{a}$ & $24.7 \mathrm{c}-\mathrm{f}$ & $42 \mathrm{ab}$ \\
\hline Margarita & $100 \mathrm{a}$ & $73 \mathrm{~b}$ & $7 \mathrm{gh}$ & $50.0 \mathrm{a}$ & $35.7 \mathrm{~b}$ & $3.5 \mathrm{i}$ & $29 \mathrm{~b}-\mathrm{f}$ \\
\hline PI 342533 & $100 \mathrm{a}$ & $100 \mathrm{a}$ & $93 \mathrm{ab}$ & $50.0 \mathrm{a}$ & $50.0 \mathrm{a}$ & $45.8 \mathrm{ab}$ & $33 \mathrm{a}-\mathrm{f}$ \\
\hline PI 358025 & $100 \mathrm{a}$ & $100 \mathrm{a}$ & $98 \mathrm{a}$ & $50.0 \mathrm{a}$ & $50.0 \mathrm{a}$ & $49.0 \mathrm{a}$ & $33 \mathrm{a}-\mathrm{f}$ \\
\hline Summer Bibb & $100 \mathrm{a}$ & $100 \mathrm{a}$ & $4 \mathrm{~h}$ & $50.0 \mathrm{a}$ & $50.0 \mathrm{a}$ & $0.4 \mathrm{i}$ & $26 \mathrm{c}-\mathrm{g}$ \\
\hline Winter Marvel & $100 \mathrm{a}$ & $0 \mathrm{~d}$ & $0 \mathrm{~h}$ & $50.0 \mathrm{a}$ & $0.0 \mathrm{e}$ & $0.0 \mathrm{i}$ & $1 \mathrm{i}$ \\
\hline
\end{tabular}

${ }^{\mathrm{z}}$ Means with the same letter in the same column are not significantly different at $P \leq 0.05$.

Table 2. Effect of temperature on seed germination in crisphead lettuce.

\begin{tabular}{|c|c|c|c|c|c|c|c|}
\hline \multirow[b]{2}{*}{ Genotype } & \multicolumn{3}{|c|}{ Germination $(\%)$} & \multicolumn{3}{|c|}{ Germination rate } & \multirow{2}{*}{$\begin{array}{c}\text { Field } \\
\text { germination }(\%)\end{array}$} \\
\hline & $24^{\circ} \mathrm{C}$ & $29^{\circ} \mathrm{C}$ & $34^{\circ} \mathrm{C}$ & $24^{\circ} \mathrm{C}$ & $29^{\circ} \mathrm{C}$ & $34^{\circ} \mathrm{C}$ & \\
\hline Barrier Reef & $99 a b^{z}$ & $87 \mathrm{ab}$ & $0 \mathrm{j}$ & $48.1 \mathrm{ab}$ & $40.7 \mathrm{bcd}$ & $0.0 \mathrm{i}$ & $5 \mathrm{jk}$ \\
\hline Batavian Cybele & $100 \mathrm{a}$ & $100 \mathrm{a}$ & $39 \mathrm{~d}-\mathrm{g}$ & $50.0 \mathrm{a}$ & $50.0 \mathrm{a}$ & $19.1 \mathrm{bcd}$ & $26 \mathrm{c}-\mathrm{f}$ \\
\hline Batavia Gloire & $100 \mathrm{a}$ & $99 \mathrm{a}$ & $71 \mathrm{ab}$ & $49.3 \mathrm{ab}$ & $46.4 \mathrm{abc}$ & $23.2 \mathrm{bc}$ & $13 \mathrm{~g}-\mathrm{j}$ \\
\hline Batavian Reine & $98 \mathrm{ab}$ & $100 \mathrm{a}$ & $56 \mathrm{bcd}$ & $48.3 \mathrm{ab}$ & $50.0 \mathrm{a}$ & $24.7 \mathrm{~b}$ & $25 \mathrm{c}-\mathrm{g}$ \\
\hline Bayview & $100 \mathrm{a}$ & $94 \mathrm{a}$ & $0 \mathrm{j}$ & $50.0 \mathrm{a}$ & $35.0 \mathrm{~d}$ & $0.0 \mathrm{i}$ & $1 \mathrm{jk}$ \\
\hline Calmar & $97 \mathrm{ab}$ & $8 \mathrm{e}$ & $2 \mathrm{j}$ & $47.3 \mathrm{~b}$ & $4.0 \mathrm{f}$ & $0.3 \mathrm{i}$ & $0 \mathrm{k}$ \\
\hline Celtic & $100 \mathrm{a}$ & $100 \mathrm{a}$ & $36 \mathrm{efg}$ & $50.0 \mathrm{a}$ & $49.0 \mathrm{a}$ & 17.0 cde & $25 \mathrm{c}-\mathrm{g}$ \\
\hline Command & $97 \mathrm{ab}$ & $95 \mathrm{a}$ & $45 \mathrm{cde}$ & $47.8 \mathrm{ab}$ & $47.3 \mathrm{ab}$ & $5.3 \mathrm{ghi}$ & $19 \mathrm{f}-\mathrm{i}$ \\
\hline Empire & $96 \mathrm{~b}$ & $100 \mathrm{a}$ & 42 def & $47.5 \mathrm{ab}$ & $50.0 \mathrm{a}$ & $13.1 \mathrm{def}$ & $21 \mathrm{e}-\mathrm{h}$ \\
\hline Headmaster & $100 \mathrm{a}$ & $100 \mathrm{a}$ & 15 hij & $50.0 \mathrm{a}$ & $50.0 \mathrm{a}$ & $7.5 \mathrm{fgh}$ & $45 \mathrm{ab}$ \\
\hline Huron & $98 \mathrm{ab}$ & $100 \mathrm{a}$ & $62 \mathrm{abc}$ & $48.5 \mathrm{ab}$ & $50.0 \mathrm{a}$ & $8.2 \mathrm{fg}$ & $28 \mathrm{c}-\mathrm{f}$ \\
\hline Marleen & $100 \mathrm{a}$ & $100 \mathrm{a}$ & 44 def & $50.0 \mathrm{a}$ & $50.0 \mathrm{a}$ & $12.0 \mathrm{ef}$ & $37 \mathrm{abc}$ \\
\hline Mid Queen & $100 \mathrm{a}$ & $100 \mathrm{a}$ & 16 hij & $50.0 \mathrm{a}$ & $50.0 \mathrm{a}$ & $8.0 \mathrm{fg}$ & $47 \mathrm{a}$ \\
\hline Premiere & $100 \mathrm{a}$ & $79 \mathrm{bc}$ & $4 j$ & $50.0 \mathrm{a}$ & $39.5 \mathrm{~cd}$ & $2.0 \mathrm{ghi}$ & $24 \mathrm{~d}-\mathrm{g}$ \\
\hline PRO 839 & $100 \mathrm{a}$ & $100 \mathrm{a}$ & $0 \mathrm{j}$ & $50.0 \mathrm{a}$ & $50.0 \mathrm{a}$ & $0.0 \mathrm{i}$ & $35 \mathrm{a}-\mathrm{d}$ \\
\hline PRO 874 & $100 \mathrm{a}$ & $100 \mathrm{a}$ & $27 \mathrm{fgh}$ & $50.0 \mathrm{a}$ & $50.0 \mathrm{a}$ & $12.9 \mathrm{def}$ & $41 \mathrm{ab}$ \\
\hline Pybas 101 & $100 \mathrm{a}$ & $100 \mathrm{a}$ & 50 cde & $47.3 \mathrm{~b}$ & $50.0 \mathrm{a}$ & $13.2 \mathrm{def}$ & $27 \mathrm{c}-\mathrm{f}$ \\
\hline Salinas & $100 \mathrm{a}$ & $43 \mathrm{~d}$ & $8 \mathrm{ij}$ & $50.0 \mathrm{a}$ & $14.6 \mathrm{e}$ & $4.0 \mathrm{ghi}$ & $23 \mathrm{~d}-\mathrm{h}$ \\
\hline Sinano-Summer & $100 \mathrm{a}$ & $100 \mathrm{a}$ & $79 \mathrm{a}$ & $50.0 \mathrm{a}$ & $50.0 \mathrm{a}$ & $37.8 \mathrm{a}$ & $35 \mathrm{a}-\mathrm{d}$ \\
\hline Vanguard 75 & $100 \mathrm{a}$ & $90 \mathrm{ab}$ & $4 \mathrm{j}$ & $50.0 \mathrm{a}$ & $45.0 \mathrm{abc}$ & $2.0 \mathrm{ghi}$ & $33 \mathrm{~b}-\mathrm{e}$ \\
\hline Vista Verde & $98 \mathrm{ab}$ & $100 \mathrm{a}$ & $0 \mathrm{j}$ & $48.5 \mathrm{ab}$ & $50.0 \mathrm{a}$ & $0.0 \mathrm{i}$ & 7 ijk \\
\hline Westlake & $100 \mathrm{a}$ & $71 \mathrm{c}$ & $0 \mathrm{j}$ & $50.0 \mathrm{a}$ & $11.9 \mathrm{e}$ & $0.0 \mathrm{i}$ & $1 \mathrm{jk}$ \\
\hline Winter Select & $100 \mathrm{a}$ & $100 \mathrm{a}$ & $3 \mathrm{j}$ & $50.0 \mathrm{a}$ & $50.0 \mathrm{a}$ & $1.5 \mathrm{hi}$ & $4 \mathrm{jk}$ \\
\hline Wintercut & $100 \mathrm{a}$ & $100 \mathrm{a}$ & $22 \mathrm{~h}$ & $50.0 \mathrm{a}$ & $50.0 \mathrm{a}$ & $8.1 \mathrm{fg}$ & $11 \mathrm{~h}-\mathrm{k}$ \\
\hline Winterhaven & $98 \mathrm{ab}$ & $47 \mathrm{~d}$ & $5 \mathrm{ij}$ & $49.0 \mathrm{ab}$ & $8.8 \mathrm{ef}$ & $2.5 \mathrm{ghi}$ & $19 \mathrm{f}-\mathrm{i}$ \\
\hline
\end{tabular}

${ }^{\mathrm{z}}$ Means with the same letter in the same column are not significantly different at $P \leq 0.05$.

Batavia Gloire $=$ Batavia Gloire du Dauphine; Batavian Reine $=$ Batavian Reine Des Glaces 
differences in the ability to germinate were observed at $34{ }^{\circ} \mathrm{C}$ (Table 1). Elizabeth, PI 358025, PI 342533, Kordaat, and Florida Buttercrisp exhibited the highest percentage of seed germination (greater than 80\%) and germination rates at $34{ }^{\circ} \mathrm{C}$. These genotypes also showed the lowest reduction in seed germination (less than $20 \%$ ) at $34{ }^{\circ} \mathrm{C}$ from that at $24{ }^{\circ} \mathrm{C}$ (Fig. 1). The results showed that these varieties were the most tolerant butterhead genotypes to thermoinhibition at $34{ }^{\circ} \mathrm{C}$. In addition to Annecy, Anthem, Dark Green Boston, and Winter Marvel, the most sensitive genotypes to thermoinhibition at $34{ }^{\circ} \mathrm{C}$ were Summer Bibb, Margarita, Aquarius, and Arcade. These genotypes exhibited a substantial reduction (greater than $80 \%$ ) in seed germination at $34^{\circ} \mathrm{C}$ (Fig. 1). Dark Green Boston has been previously considered a thermosensitive cultivar, which was consistent with our results (Sung et al., 2008).

Seed germination in the field was low with the highest germination percentages (greater than 30\%) observed in Belluro, Mantilia, Aquarius, Kordaat, Magnet, PI 342533, PI 358025, Arcade, and Kitty (Table 1). There was a significant positive correlation between seed germination at 29 and $34{ }^{\circ} \mathrm{C}$ and field germination in butterhead lettuce (Table 7). Thus, seed germination of some lettuce genotypes is inhibited at $29^{\circ} \mathrm{C}$, but others can tolerate higher temperatures $\left(34^{\circ} \mathrm{C}\right)$, which indicated that the maximum temperature for seed germination is genotype-dependent (Coons et al., 1990).

Crisphead lettuce. All crisphead genotypes showed a high percentage germination (greater than 95\%) and germination rate at $24{ }^{\circ} \mathrm{C}$ (Table 2 ). There were significant differences in percentage germination and germination rate among all genotypes at higher temperatures. At $29^{\circ} \mathrm{C}$, most crisphead genotypes germinated well except Calmar, Salinas, and Winterhaven, which showed the lowest germination percentage $(8 \%, 43 \%$, and $47 \%$, respectively). These genotypes also showed the largest reduction (greater than 50\%) in seed germination at $29^{\circ} \mathrm{C}$ (Fig. 2). The results indicated that Calmar was the most thermosensitive genotype followed by Salinas and Winterheaven. At $34{ }^{\circ} \mathrm{C}$, Sinano-Summer, Batavia Gloire du Dauphine, Huron, and Batavian Reine Des Glaces showed the highest germination percentages $(79 \%, 71 \%, 62 \%$, $56 \%$, respectively) among crisphead genotypes. These genotypes also showed the highest germination rates at $34{ }^{\circ} \mathrm{C}$ (Table 2) and the smallest reduction (less than 50\%) in germination at $34{ }^{\circ} \mathrm{C}$ (Fig. 2). All the other genotypes had lower germination and some of them were completely inhibited with no germination at $34{ }^{\circ} \mathrm{C}$ such as Barrier Reef, Bayview, Pro 839, Vista Verde, and Westlake. SinanoSummer was the only crisphead lettuce with low reduction in germination at $34{ }^{\circ} \mathrm{C}$ and it was considered the most thermotolerant genotype (Fig. 2). Salinas exhibited thermoinhibition at 29 and $34{ }^{\circ} \mathrm{C}$ and this result was consistent with the previously reported findings (Argyris et al., 2008a, 2008b; Coons et al., 1990).

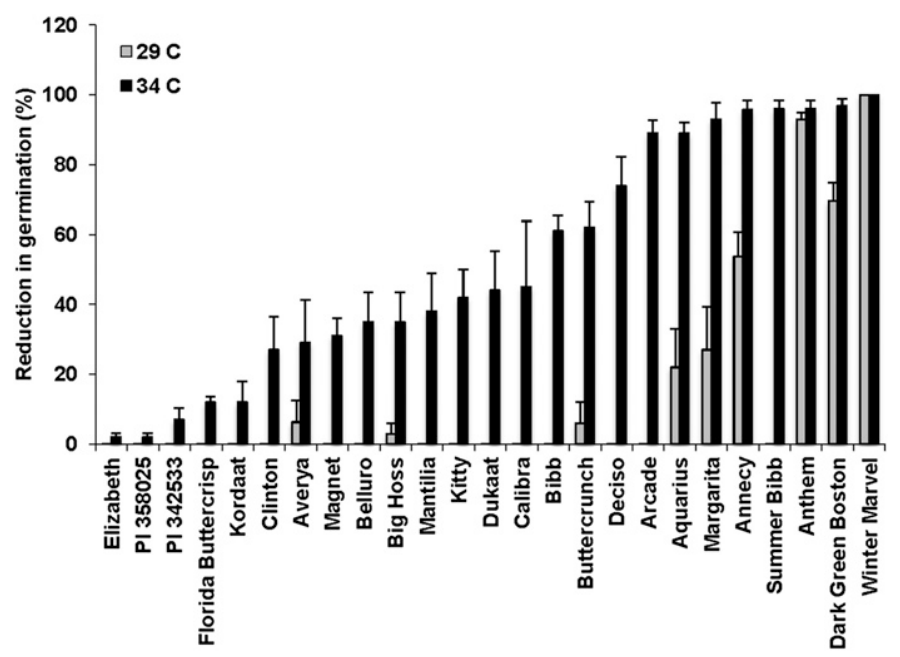

Fig. 1. Reduction in germination percentage at 29 and $34{ }^{\circ} \mathrm{C}$ from $24{ }^{\circ} \mathrm{C}$ in butterhead lettuce. Results are means $\pm \operatorname{SE}(\mathrm{n}=4) . \mathrm{LSD}_{0.05}$ for 29 and $34{ }^{\circ} \mathrm{C}$ are 11.9 and 20.9 , respectively. LSD $=$ least significant difference.

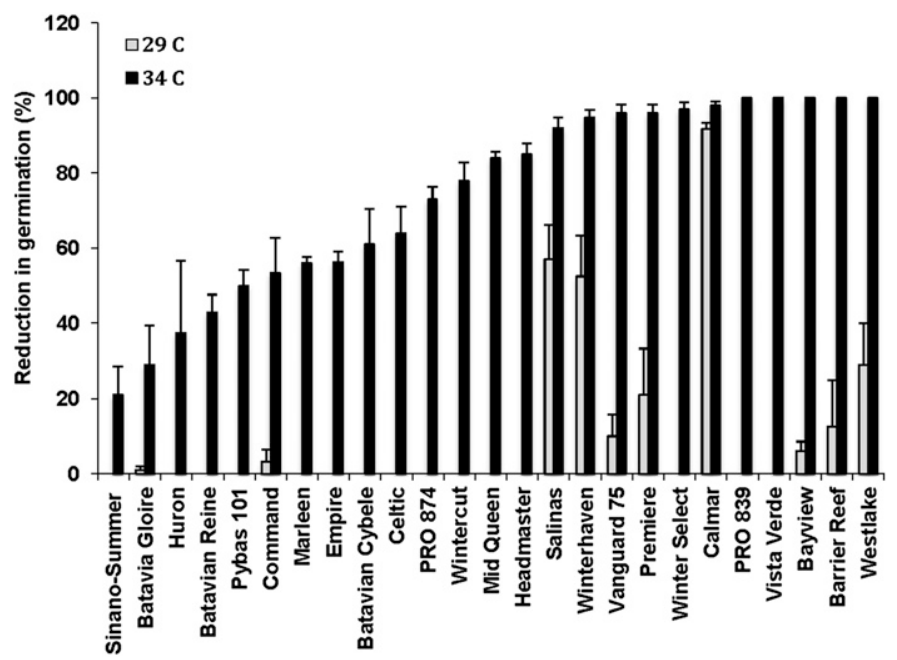

Fig. 2. Reduction in germination percentage at 29 and $34{ }^{\circ} \mathrm{C}$ from $24{ }^{\circ} \mathrm{C}$ in crisphead lettuce. Results are means $\pm S E(n=4) . L^{2 S D} D_{05}$ for 29 and $34{ }^{\circ} \mathrm{C}$ are 14.8 and 16.7 , respectively. $L S D=$ least significant difference.

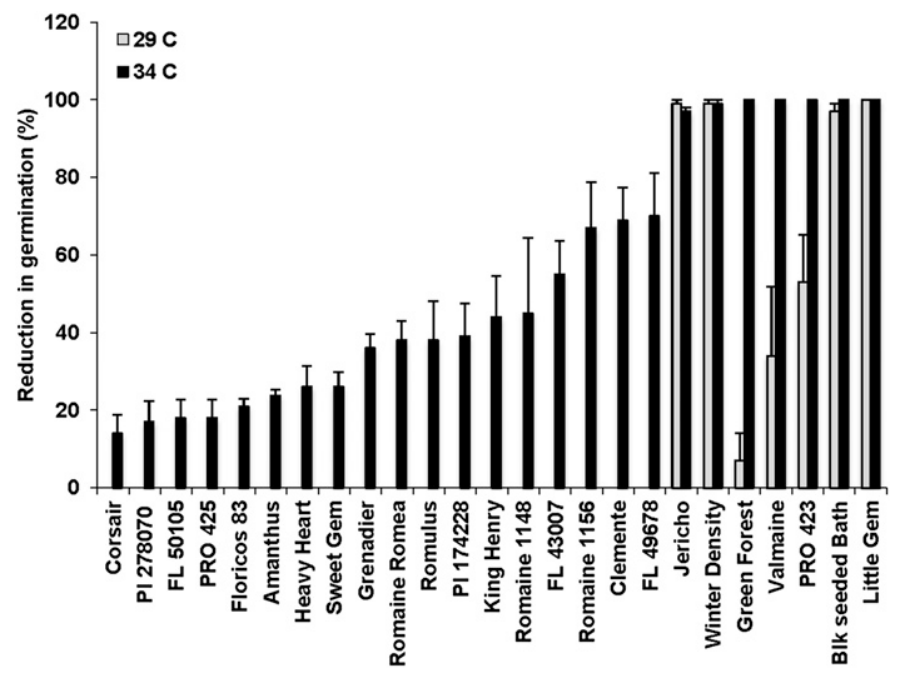

Fig. 3. Reduction in germination percentage at 29 and $34{ }^{\circ} \mathrm{C}$ from $24{ }^{\circ} \mathrm{C}$ in romaine lettuce. Results are means $\pm \mathrm{SE}(\mathrm{n}=4) . \mathrm{LSD}_{0.05}$ for 29 and $34{ }^{\circ} \mathrm{C}$ are 12.8 and 20.2 , respectively. LSD = least significant difference. 
Table 3. Effect of temperature on seed germination in romaine lettuce.

\begin{tabular}{|c|c|c|c|c|c|c|c|}
\hline \multirow[b]{2}{*}{ Genotype } & \multicolumn{3}{|c|}{ Germination $(\%)$} & \multicolumn{3}{|c|}{ Germination rate } & \multirow{2}{*}{$\begin{array}{c}\text { Field } \\
\text { germination }(\%)\end{array}$} \\
\hline & $24{ }^{\circ} \mathrm{C}$ & $29^{\circ} \mathrm{C}$ & $34{ }^{\circ} \mathrm{C}$ & $24{ }^{\circ} \mathrm{C}$ & $29^{\circ} \mathrm{C}$ & $34{ }^{\circ} \mathrm{C}$ & \\
\hline Amanthus & $93 b^{z}$ & $100 \mathrm{a}$ & $71 \mathrm{a}-\mathrm{e}$ & $46.5 \mathrm{~b}$ & $50.0 \mathrm{a}$ & $35.3 \mathrm{ab}$ & $11 \mathrm{ijk}$ \\
\hline Black Seeded Bath & $100 \mathrm{a}$ & $3 \mathrm{~d}$ & $0 \mathrm{~h}$ & $50.0 \mathrm{a}$ & $1.5 \mathrm{~d}$ & $0.0 \mathrm{~g}$ & $2 \mathrm{jk}$ \\
\hline Clemente & $100 \mathrm{a}$ & $100 \mathrm{a}$ & $31 \mathrm{~g}$ & $50.0 \mathrm{a}$ & $49.0 \mathrm{a}$ & $14.8 \mathrm{ef}$ & $13 \mathrm{ijk}$ \\
\hline Corsair & $100 \mathrm{a}$ & $100 \mathrm{a}$ & $86 \mathrm{a}$ & $50.0 \mathrm{a}$ & $50.0 \mathrm{a}$ & $39.7 \mathrm{a}$ & $55 \mathrm{ab}$ \\
\hline FL 43007 & $100 \mathrm{a}$ & $100 \mathrm{a}$ & $45 \mathrm{fg}$ & $50.0 \mathrm{a}$ & $50.0 \mathrm{a}$ & $18.6 \mathrm{def}$ & $41 \mathrm{c}-\mathrm{f}$ \\
\hline FL 49678 & $100 \mathrm{a}$ & $100 \mathrm{a}$ & $30 \mathrm{~g}$ & $50.0 \mathrm{a}$ & $50.0 \mathrm{a}$ & $13.0 \mathrm{f}$ & $39 \mathrm{c}-\mathrm{f}$ \\
\hline FL 50105 & $100 \mathrm{a}$ & $100 \mathrm{a}$ & $82 \mathrm{abc}$ & $50.0 \mathrm{a}$ & $50.0 \mathrm{a}$ & $37.2 \mathrm{a}$ & $57 \mathrm{a}$ \\
\hline Floricos 83 & $100 \mathrm{a}$ & $100 \mathrm{a}$ & $79 \mathrm{a}-\mathrm{d}$ & $50.0 \mathrm{a}$ & $50.0 \mathrm{a}$ & $35.6 \mathrm{ab}$ & $40 \mathrm{c}-\mathrm{f}$ \\
\hline Green Forest & $100 \mathrm{a}$ & $93 \mathrm{a}$ & $0 \mathrm{~h}$ & $50.0 \mathrm{a}$ & $46.1 \mathrm{a}$ & $0.0 \mathrm{~g}$ & $43 \mathrm{~b}-\mathrm{e}$ \\
\hline Grenadier & $100 \mathrm{a}$ & $100 \mathrm{a}$ & $64 b-f$ & $50.0 \mathrm{a}$ & $50.0 \mathrm{a}$ & $31.4 \mathrm{abc}$ & $43 \mathrm{~b}-\mathrm{e}$ \\
\hline Heavy Heart & $100 \mathrm{a}$ & $100 \mathrm{a}$ & $74 \mathrm{a}-\mathrm{e}$ & $50.0 \mathrm{a}$ & $50.0 \mathrm{a}$ & $36.4 \mathrm{ab}$ & $32 \mathrm{~d}-\mathrm{g}$ \\
\hline Jericho & $100 \mathrm{a}$ & $1 \mathrm{~d}$ & $3 \mathrm{~h}$ & $50.0 \mathrm{a}$ & $0.5 \mathrm{~d}$ & $0.4 \mathrm{~g}$ & $1 \mathrm{k}$ \\
\hline King Henry & $98 \mathrm{a}$ & $100 \mathrm{a}$ & 55 ef & $49.0 \mathrm{a}$ & $50.0 \mathrm{a}$ & $27.3 \mathrm{bcd}$ & 31 efg \\
\hline Little Gem & $100 \mathrm{a}$ & $0 \mathrm{~d}$ & $0 \mathrm{~h}$ & $50.0 \mathrm{a}$ & $0.0 \mathrm{~d}$ & $0.0 \mathrm{~g}$ & $7 \mathrm{jk}$ \\
\hline PI 174228 & $100 \mathrm{a}$ & $100 \mathrm{a}$ & $61 \mathrm{def}$ & $50.0 \mathrm{a}$ & $50.0 \mathrm{a}$ & 23.9 cde & $28 \mathrm{fgh}$ \\
\hline PI $278070 \mathrm{BS}$ & $100 \mathrm{a}$ & $100 \mathrm{a}$ & $83 \mathrm{ab}$ & $50.0 \mathrm{a}$ & $50.0 \mathrm{a}$ & $38.9 \mathrm{a}$ & 30 efg \\
\hline PRO 423 & $100 \mathrm{a}$ & $47 \mathrm{c}$ & $0 \mathrm{~h}$ & $50.0 \mathrm{a}$ & $23.5 \mathrm{c}$ & $0.0 \mathrm{~g}$ & $15 \mathrm{hij}$ \\
\hline PRO 425 & $100 \mathrm{a}$ & $100 \mathrm{a}$ & $82 \mathrm{abc}$ & $50.0 \mathrm{a}$ & $50.0 \mathrm{a}$ & $40.3 \mathrm{a}$ & $57 \mathrm{a}$ \\
\hline Romaine Romea & $100 \mathrm{a}$ & $100 \mathrm{a}$ & $62 \mathrm{c}-\mathrm{f}$ & $50.0 \mathrm{a}$ & $50.0 \mathrm{a}$ & $31.0 \mathrm{abc}$ & $45 \mathrm{a}-\mathrm{d}$ \\
\hline Romaine SSC 1148 & $100 \mathrm{a}$ & $100 \mathrm{a}$ & 55 ef & $50.0 \mathrm{a}$ & $50.0 \mathrm{a}$ & $27.3 \mathrm{bcd}$ & $48 \mathrm{abc}$ \\
\hline Romaine SSC 1156 & $100 \mathrm{a}$ & $100 \mathrm{a}$ & $33 \mathrm{~g}$ & $50.0 \mathrm{a}$ & $50.0 \mathrm{a}$ & $15.5 \mathrm{ef}$ & $37 \mathrm{c}-\mathrm{f}$ \\
\hline Romulus & $100 \mathrm{a}$ & $100 \mathrm{a}$ & $62 \mathrm{c}-\mathrm{f}$ & $50.0 \mathrm{a}$ & $50.0 \mathrm{a}$ & $27.4 \mathrm{bcd}$ & $21 \mathrm{ghi}$ \\
\hline Sweet Gem & $100 \mathrm{a}$ & $100 \mathrm{a}$ & $74 \mathrm{a}-\mathrm{e}$ & $50.0 \mathrm{a}$ & $50.0 \mathrm{a}$ & $36.5 \mathrm{ab}$ & $37 \mathrm{c}-\mathrm{f}$ \\
\hline Valmaine & $100 \mathrm{a}$ & $66 \mathrm{~b}$ & $0 \mathrm{~h}$ & $50.0 \mathrm{a}$ & $32.1 \mathrm{~b}$ & $0.0 \mathrm{~g}$ & $2 \mathrm{jk}$ \\
\hline Winter Density & $100 \mathrm{a}$ & $1 \mathrm{~d}$ & $1 \mathrm{~h}$ & $50.0 \mathrm{a}$ & $0.3 \mathrm{~d}$ & $0.5 \mathrm{~g}$ & $2 \mathrm{jk}$ \\
\hline
\end{tabular}

${ }^{2}$ Means with the same letter in the same column are not significantly different at $P \leq 0.05$.

Table 4. Effect of temperature on seed germination in green leaf lettuce.

\begin{tabular}{|c|c|c|c|c|c|c|c|}
\hline \multirow[b]{2}{*}{ Genotype } & \multicolumn{3}{|c|}{ Germination $(\%)$} & \multicolumn{3}{|c|}{ Germination rate } & \multirow{2}{*}{$\begin{array}{c}\text { Field } \\
\text { germination }(\%)\end{array}$} \\
\hline & $24^{\circ} \mathrm{C}$ & $29^{\circ} \mathrm{C}$ & $34^{\circ} \mathrm{C}$ & $24^{\circ} \mathrm{C}$ & $29^{\circ} \mathrm{C}$ & $34^{\circ} \mathrm{C}$ & \\
\hline Amazona & $100 \mathrm{a}^{\mathrm{z}}$ & $99 \mathrm{a}$ & $41 \mathrm{~d}-\mathrm{g}$ & $50.0 \mathrm{a}$ & $35.9 \mathrm{c}$ & 19.8 cde & $8 \mathrm{ghi}$ \\
\hline Antigua & $98 \mathrm{a}$ & $100 \mathrm{a}$ & $41 \mathrm{~d}-\mathrm{g}$ & $41.4 \mathrm{~b}$ & $50.0 \mathrm{a}$ & 19.2 cde & $14 \mathrm{e}-\mathrm{h}$ \\
\hline Australischer Gelber & $95 \mathrm{~b}$ & $30 \mathrm{e}$ & $0 \mathrm{k}$ & $41.3 \mathrm{~b}$ & $7.6 \mathrm{f}$ & $0.0 \mathrm{~h}$ & 5 hi \\
\hline Azura & $100 \mathrm{a}$ & $92 \mathrm{ab}$ & $56 \mathrm{~b}-\mathrm{e}$ & $49.5 \mathrm{a}$ & $45.0 \mathrm{ab}$ & $22.5 \mathrm{bcd}$ & $5 \mathrm{hi}$ \\
\hline Black Seeded Simpson & $100 \mathrm{a}$ & $87 \mathrm{ab}$ & $1 \mathrm{k}$ & $50.0 \mathrm{a}$ & $39.6 \mathrm{bc}$ & $0.5 \mathrm{~h}$ & $4 \mathrm{hi}$ \\
\hline Flandria & $100 \mathrm{a}$ & 100 & $29 \mathrm{fgh}$ & $50.0 \mathrm{a}$ & $50.0 \mathrm{a}$ & 13.9 def & $5 \mathrm{hi}$ \\
\hline Funly & $100 \mathrm{a}$ & ND & $28 \mathrm{gh}$ & $50.0 \mathrm{a}$ & ND & $12.2 \mathrm{efg}$ & $28 \mathrm{~cd}$ \\
\hline Green Wave & $100 \mathrm{a}$ & $100 \mathrm{a}$ & 50 cde & $50.0 \mathrm{a}$ & $50.0 \mathrm{a}$ & $22.8 \mathrm{bc}$ & $42 \mathrm{ab}$ \\
\hline Greengo & $98 \mathrm{a}$ & $49 \mathrm{~d}$ & $14 \mathrm{~h}-\mathrm{k}$ & $38.3 \mathrm{~b}$ & $12.1 \mathrm{ef}$ & $1.2 \mathrm{~h}$ & $0 \mathrm{i}$ \\
\hline Loros & $100 \mathrm{a}$ & $100 \mathrm{a}$ & $26 \mathrm{gh}$ & $50.0 \mathrm{a}$ & $50.0 \mathrm{a}$ & $4.0 \mathrm{gh}$ & $20 \mathrm{~d}-\mathrm{g}$ \\
\hline Neva & $100 \mathrm{a}$ & $100 \mathrm{a}$ & 49 def & $50.0 \mathrm{a}$ & $50.0 \mathrm{a}$ & $23.8 \mathrm{bc}$ & $27 \mathrm{~cd}$ \\
\hline Noemie & $100 \mathrm{a}$ & $100 \mathrm{a}$ & $98 \mathrm{a}$ & $50.0 \mathrm{a}$ & $50.0 \mathrm{a}$ & $47.5 \mathrm{a}$ & $42 \mathrm{ab}$ \\
\hline Ocean Green & $100 \mathrm{a}$ & $100 \mathrm{a}$ & $5 \mathrm{ijk}$ & $50.0 \mathrm{a}$ & $50.0 \mathrm{a}$ & $1.7 \mathrm{~h}$ & $21 \mathrm{def}$ \\
\hline PI 177420 & $100 \mathrm{a}$ & $100 \mathrm{a}$ & $70 \mathrm{bc}$ & $50.0 \mathrm{a}$ & $50.0 \mathrm{a}$ & $26.1 \mathrm{bc}$ & 27 cde \\
\hline PI 187238 E & $100 \mathrm{a}$ & $79 \mathrm{bc}$ & $73 \mathrm{~b}$ & $50.0 \mathrm{a}$ & $25.4 \mathrm{~d}$ & $30.2 \mathrm{~b}$ & $36 \mathrm{abc}$ \\
\hline Royal Oak Leaf & $100 \mathrm{a}$ & $11 \mathrm{f}$ & $14 \mathrm{~h}-\mathrm{k}$ & $50.0 \mathrm{a}$ & $5.0 \mathrm{f}$ & $3.1 \mathrm{~h}$ & 3 hi \\
\hline Simpson Elite & $100 \mathrm{a}$ & $31 \mathrm{e}$ & $0 \mathrm{k}$ & $50.0 \mathrm{a}$ & $15.5 \mathrm{e}$ & $0.0 \mathrm{~h}$ & 8 ghi \\
\hline Slobolt & $100 \mathrm{a}$ & $69 \mathrm{c}$ & 2 jk & $46.5 \mathrm{a}$ & $27.1 \mathrm{~d}$ & $0.6 \mathrm{~h}$ & $10 \mathrm{f}-\mathrm{i}$ \\
\hline Squadron & $100 \mathrm{a}$ & $19 \mathrm{ef}$ & $12 \mathrm{~h}-\mathrm{k}$ & $48.5 \mathrm{a}$ & $7.0 \mathrm{f}$ & $6.0 \mathrm{fgh}$ & $44 \mathrm{a}$ \\
\hline Tendergreen & $100 \mathrm{a}$ & $100 \mathrm{a}$ & 38 efg & $50.0 \mathrm{a}$ & $50.0 \mathrm{a}$ & $6.6 \mathrm{fgh}$ & $19 \mathrm{~d}-\mathrm{g}$ \\
\hline Tiara & $100 \mathrm{a}$ & $100 \mathrm{a}$ & $22 \mathrm{~g}-\mathrm{j}$ & $50.0 \mathrm{a}$ & $50.0 \mathrm{a}$ & $6.7 \mathrm{fgh}$ & $30 \mathrm{bcd}$ \\
\hline Two Star & $100 \mathrm{a}$ & $100 \mathrm{a}$ & $0 \mathrm{k}$ & $50.0 \mathrm{a}$ & $50.0 \mathrm{a}$ & $0.0 \mathrm{~h}$ & 5 hi \\
\hline Versailles & $100 \mathrm{a}$ & $100 \mathrm{a}$ & $23 \mathrm{ghi}$ & $50.0 \mathrm{a}$ & $50.0 \mathrm{a}$ & $8.4 \mathrm{fgh}$ & 5 hi \\
\hline Waldmann's Green & $100 \mathrm{a}$ & $72 \mathrm{c}$ & $14 \mathrm{~h}-\mathrm{k}$ & $50.0 \mathrm{a}$ & $34.7 \mathrm{c}$ & $7.0 \mathrm{fgh}$ & 24 cde \\
\hline Xena & $100 \mathrm{a}$ & $100 \mathrm{a}$ & $61 \mathrm{bcd}$ & $50.0 \mathrm{a}$ & $50.0 \mathrm{a}$ & $30.1 \mathrm{~b}$ & $44 \mathrm{a}$ \\
\hline
\end{tabular}

${ }^{\mathrm{z}}$ Means with the same letter in the same column are not significantly different at $P \leq 0.05$.

$\mathrm{ND}=$ not determined

The highest germination percentages (greater than 30\%) in the field were observed in Mid Queen, Headmaster, PRO 874, Marleen, PRO 839, Sinano-Summer, and Vanguard 75 (Table 2). There was no significant correlation between field germination and germination at 29 or $34^{\circ} \mathrm{C}$ among crisphead genotypes (Table 7).

Romaine lettuce. Germination percentage and germination rates for romaine lettuce genotypes are shown in Table 3. There were significant differences in percentage germination and germination rates among genotypes at 29 and $34{ }^{\circ} \mathrm{C}$. Seeds of Little Gem, Jericho, Winter Density, and Black Seeded Bath exhibited thermoinhibition at $29{ }^{\circ} \mathrm{C}$ as indicated by the lowest germination percentages and germination rates of the romaine genotypes tested. These genotypes also showed the largest reduction in seed germination at $29^{\circ} \mathrm{C}$ compared with other genotypes (Fig. 3). In addition to these genotypes, Green Forest, Valmaine, and PRO 423 exhibited substantial reductions in seed germination at $34{ }^{\circ} \mathrm{C}$
(Fig. 3). These genotypes were more sensitive to thermoinhibition at $34{ }^{\circ} \mathrm{C}$ than the other genotypes. The most thermotolerant genotypes were Corsair, PI 278070, FL. 50105, Pro 425, Floricos 83, Amanthus, Heavy Heart, and Sweet Gem, which showed the smallest reduction in seed germination (less than $30 \%$ ) at $34{ }^{\circ} \mathrm{C}$ compared with other romaine genotypes (Fig. 3). Green Forest exhibited thermotolerance at $29{ }^{\circ} \mathrm{C}$ and thermoinhibition at $34{ }^{\circ} \mathrm{C}$. This indicated that the maximum temperature for thermoinhibition depends on lettuce cultivar and germplasm.

The highest field germination percentages (greater than $50 \%$ ) were observed in PRO 425, FL 50105, and Corsair (Table 3). The field tolerance of these genotypes was consistent with their thermotolerance at 29 and $34{ }^{\circ} \mathrm{C}$. There was a significant positive correlation between field seed germination and germination at 29 and $34{ }^{\circ} \mathrm{C}$ among romaine genotypes (Table 7).

Green leaf lettuce. The effect of temperature on seed germination percentage and germination rate in green leaf lettuce is presented in Table 4. High germination percentages (greater than 95\%) were observed in all genotypes at $24^{\circ} \mathrm{C}$. At 29 and $34^{\circ} \mathrm{C}$, there were significant differences in percentage germination and germination rates among green leaf genotypes. The most sensitive genotypes to thermoinhibition were Royal Oak Leaf, Squadron, Australischer Gelber, Simpson Elite, and Greengo, which had significantly lower germination percentages (less than $50 \%$ ) and germination rates at $29^{\circ} \mathrm{C}$ than other green leaf genotypes (Table 4). The reduction in germination percentages at $29{ }^{\circ} \mathrm{C}$ in these genotypes was $89 \%, 81 \%, 69 \%, 69 \%$, and $50 \%$, respectively (Fig. 4). The differences in germination percentages and germination rates among lettuce genotypes were greater at $34{ }^{\circ} \mathrm{C}$ than at $29^{\circ} \mathrm{C}$. Noemie was the most thermotolerant genotype followed by PI $187238 \mathrm{E}$ and PI 177420. These genotypes had the highest germination percentages and germination rates at $34^{\circ} \mathrm{C}$ as compared with the other genotypes (Table 4 ). The reductions in seed germination in these genotypes at $34{ }^{\circ} \mathrm{C}$ were $2 \%, 27 \%$, and $30 \%$, respectively (Fig. 4).

The largest field germination percentages (greater than 40\%) were observed in Squadron, Xena, Noemie, and Green Wave (Table 4). There was a significant correlation between field seed germination and germination at $34{ }^{\circ} \mathrm{C}$ among green leaf genotypes (Table 7).

Red leaf lettuce. Cultivars and genotypes of red leaf lettuce also differ in their tolerance to thermoinhibition. All red leaf lettuce genotypes exhibited high germination percentages and germination rates at $24{ }^{\circ} \mathrm{C}$ (Table 5). However, at 29 and $34{ }^{\circ} \mathrm{C}$, there were highly significant differences in germination percentages and germination rates among genotypes. At $29^{\circ} \mathrm{C}$, most of the red lettuce genotypes exhibited high germination percentages and rates except Big Red, Red Flower, Prizehead, Red Rage, Red Prize, Ibis, Merlot, Red Tide, and Hyper Red Rumple Waved, which showed the lowest germination 


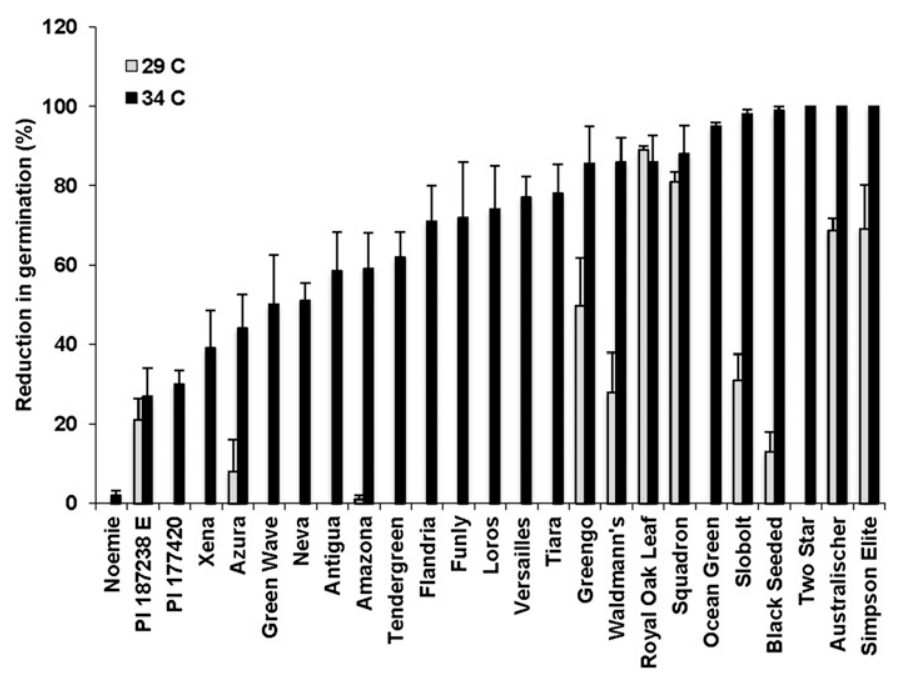

Fig. 4. Reduction in germination percentage at 29 and $34^{\circ} \mathrm{C}$ from $24^{\circ} \mathrm{C}$ in green leaf lettuce. Results are means $\pm \operatorname{SE}(\mathrm{n}=4)$. $\mathrm{LSD}_{0.05}$ for 29 and $34^{\circ} \mathrm{C}$ are 13.7 and 20.6, respectively. Black Seeded $=$ Black Seeded Simpson. LSD $=$ least significant difference.

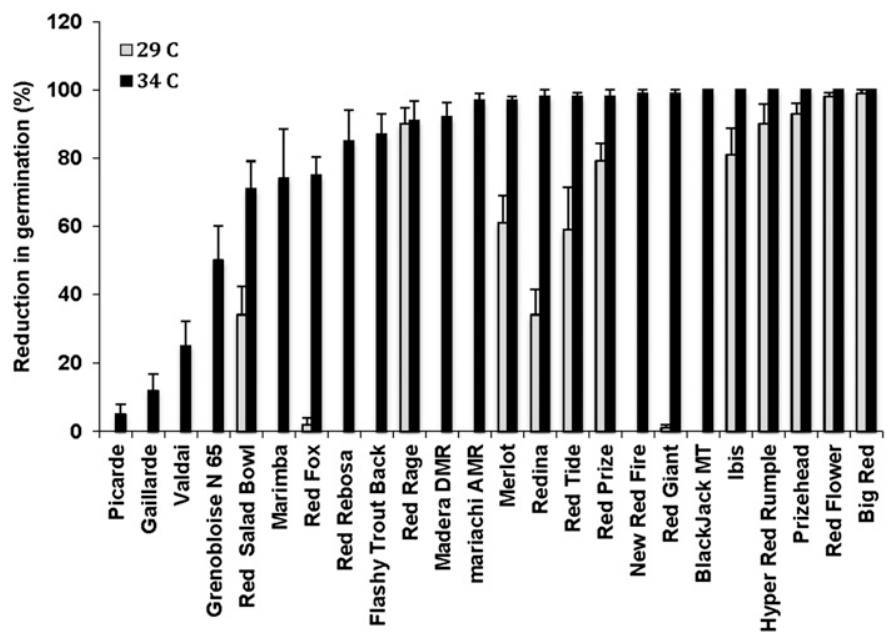

Fig. 5. Reduction in germination percentage at 29 and $34{ }^{\circ} \mathrm{C}$ from $24{ }^{\circ} \mathrm{C}$ in red leaf lettuce. Results are means $\pm \operatorname{SE}(\mathrm{n}=4) . \mathrm{LSD}_{0.05}$ for 29 and $34{ }^{\circ} \mathrm{C}$ are 12.9 and 15.1 , respectively. $\mathrm{LSD}=$ least significant difference.

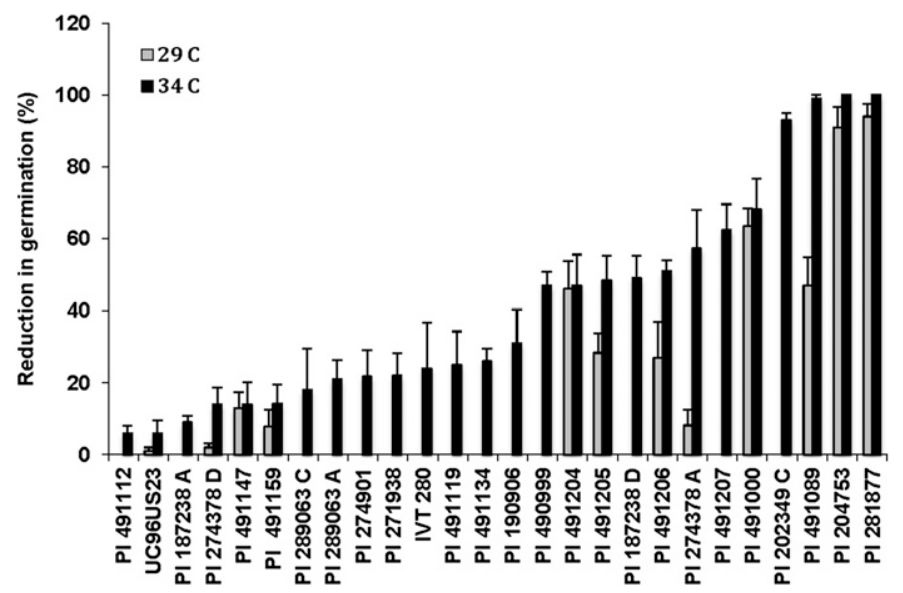

Fig. 6. Reduction in germination percentage at 29 and $34{ }^{\circ} \mathrm{C}$ from $24{ }^{\circ} \mathrm{C}$ in wild species. Results are means $\pm \mathrm{SE}(\mathrm{n}=4) . \mathrm{LSD}_{0.05}$ for 29 and $34^{\circ} \mathrm{C}$ are 10.8 and 18.3 , respectively. $\mathrm{LSD}=$ least significant difference. percentages and rates. However, at $34{ }^{\circ} \mathrm{C}$, most of the genotypes showed very low germination percentage and rates except Picarde, Gaillarde, and Valdai (Table 5). The reduction in germination percentages at $34^{\circ} \mathrm{C}$ from that at $24{ }^{\circ} \mathrm{C}$ in Picarde, Gaillarde, and Valdai was $5 \%, 12 \%$, and $25 \%$, respectively (Fig. 5). These red leaf genotypes were considered the most tolerant to thermoinhibition at $34{ }^{\circ} \mathrm{C}$.

The highest field germination percentages (greater than 30\%) were observed in Picarde, Red Giant, Medera DMR, Marimba, Grenobloise N 65, Gaillarde, and Black Jack (Table 5). Picarde showed the highest germination in the field and at $34{ }^{\circ} \mathrm{C}$. There was a significant positive correlation between field seed germination and germination at 29 and $34{ }^{\circ} \mathrm{C}$ among red leaf genotypes (Table 7).

Wild species. There were significant differences in germination percentages and rates among primitive and wild lettuce species at 29 and $34{ }^{\circ} \mathrm{C}$ (Table 6). PI 281877, PI 204753, and PI 491000 had the lowest germination percentages and rates at $29^{\circ} \mathrm{C}$. The reduction in germination percentages in PI 281877, PI 204753, and PI 491000 at $29^{\circ} \mathrm{C}$ was $94 \%, 91 \%$, and 63 , respectively (Fig. 6). These genotypes were the most thermosensitive genotypes at $29{ }^{\circ} \mathrm{C}$. In addition to these genotypes, PI 491089 and PI 202349 C exhibited thermoinhibition at $34{ }^{\circ} \mathrm{C}$ as determined by germination percentages and rates (Table 6). Genotypes with high tolerance to thermoinhibition were PI 491112, UC96US23, PI 187238 A, PI 274378 D, PI 491147, and PI $289063 \mathrm{C}$ as indicated by high germination percentages (greater than $80 \%$ ) and germination rates at $34{ }^{\circ} \mathrm{C}$ (Table 6). The reductions in seed germination in these genotypes were $6 \%, 6 \%, 9 \%, 14 \%, 14 \%$, and $18 \%$, respectively (Fig. 6). This shows that all three wild species (L. serriola, L. saligna, and L. virosa) and primitive lettuce have thermoinsensitive genotypes. The observed thermotolerance in UC96US23 (Lactuca serriola) was consistent with previous results of Argyris et al. (2008a, 2008b).

All of the wild species showed very low germination in the field (Table 6). The highest percentage germination (33\%) was observed in PI 289063 A. There was no significant correlation between field seed germination and germination at 29 or $34{ }^{\circ} \mathrm{C}$ among wild species (Table 7). This variation in seed germination could be the result of field conditions, which were not optimal for germination like in the laboratory or growth chambers. Disease and insect damage may also lower germination percentage. It has been observed that poor field emergence at high temperature in broccoli was the result of an inhibition of root growth (Jett et al., 1996). The presence of high salt in the irrigation water or in the upper soil surface may lower seed germination (Coons et al., 1990).

The observed variations in the sensitivity to high-temperature inhibition of seed germination among lettuce genotypes may depend on lettuce cultivars and germplasm (Coons et al., 1990; Gray, 1975; Thompson et al., 1979). The regulation of seed germination in 
Table 5. Effect of temperature on seed germination in red leaf lettuce.

\begin{tabular}{|c|c|c|c|c|c|c|c|}
\hline \multirow[b]{2}{*}{ Genotype } & \multicolumn{3}{|c|}{ Germination (\%) } & \multicolumn{3}{|c|}{ Germination rate } & \multirow{2}{*}{$\begin{array}{c}\text { Field } \\
\text { germination }(\%)\end{array}$} \\
\hline & $24^{\circ} \mathrm{C}$ & $29^{\circ} \mathrm{C}$ & $34^{\circ} \mathrm{C}$ & $24^{\circ} \mathrm{C}$ & $29^{\circ} \mathrm{C}$ & $34^{\circ} \mathrm{C}$ & \\
\hline Big Red & $89 b^{z}$ & $1 \mathrm{e}$ & $0 \mathrm{f}$ & $37.1 \mathrm{~b}$ & $0.3 \mathrm{~g}$ & $0.0 \mathrm{e}$ & $5 \mathrm{jk}$ \\
\hline BlackJack MT & $100 \mathrm{a}$ & $100 \mathrm{a}$ & $0 \mathrm{f}$ & $50.0 \mathrm{a}$ & $50.0 \mathrm{a}$ & $0.0 \mathrm{e}$ & $30 \mathrm{c}-\mathrm{f}$ \\
\hline Flashy Trout Back & $100 \mathrm{a}$ & $100 \mathrm{a}$ & $13 \mathrm{ef}$ & $50.0 \mathrm{a}$ & $50.0 \mathrm{a}$ & $6.1 \mathrm{de}$ & $22 \mathrm{e}-\mathrm{h}$ \\
\hline Gaillarde & $100 \mathrm{a}$ & $100 \mathrm{a}$ & $88 \mathrm{ab}$ & $50.0 \mathrm{a}$ & $50.0 \mathrm{a}$ & $43.5 \mathrm{a}$ & $33 \mathrm{~b}-\mathrm{e}$ \\
\hline Grenobloise N 65 & $100 \mathrm{a}$ & $100 \mathrm{a}$ & $50 \mathrm{c}$ & $50.0 \mathrm{a}$ & $50.0 \mathrm{a}$ & $23.4 \mathrm{c}$ & $34 \mathrm{~b}-\mathrm{e}$ \\
\hline Hyper Red Rumple & $99 \mathrm{a}$ & $10 \mathrm{de}$ & $0 \mathrm{f}$ & $49.5 \mathrm{a}$ & $1.2 \mathrm{fg}$ & $0.0 \mathrm{e}$ & $2 \mathrm{k}$ \\
\hline Ibis & $100 \mathrm{a}$ & $19 \mathrm{~d}$ & $0 \mathrm{f}$ & $50.0 \mathrm{a}$ & $7.6 \mathrm{ef}$ & $0.0 \mathrm{e}$ & $0 \mathrm{k}$ \\
\hline Madera DMR & $100 \mathrm{a}$ & $100 \mathrm{a}$ & $8 \mathrm{f}$ & $50.0 \mathrm{a}$ & $50.0 \mathrm{a}$ & $1.9 \mathrm{e}$ & $39 \mathrm{abc}$ \\
\hline Mariachi AMR & $100 \mathrm{a}$ & $100 \mathrm{a}$ & $3 \mathrm{f}$ & $50.0 \mathrm{a}$ & $50.0 \mathrm{a}$ & $1.5 \mathrm{e}$ & 8 ijk \\
\hline Marimba & $100 \mathrm{a}$ & $100 \mathrm{a}$ & $26 \mathrm{de}$ & $50.0 \mathrm{a}$ & $50.0 \mathrm{a}$ & $11.1 \mathrm{~d}$ & $37 \mathrm{a}-\mathrm{d}$ \\
\hline Merlot & $100 \mathrm{a}$ & $39 \mathrm{c}$ & $3 \mathrm{f}$ & $50.0 \mathrm{a}$ & $19.5 \mathrm{~cd}$ & $1.5 \mathrm{e}$ & $11 \mathrm{~h}-\mathrm{k}$ \\
\hline New Red Fire & $100 \mathrm{a}$ & $100 \mathrm{a}$ & $1 \mathrm{f}$ & $50.0 \mathrm{a}$ & $50.0 \mathrm{a}$ & $0.5 \mathrm{e}$ & $25 \mathrm{~d}-\mathrm{g}$ \\
\hline Picarde & $100 \mathrm{a}$ & $100 \mathrm{a}$ & $95 \mathrm{a}$ & $50.0 \mathrm{a}$ & $50.0 \mathrm{a}$ & $46.8 \mathrm{a}$ & $49 \mathrm{a}$ \\
\hline Prizehead & $100 \mathrm{a}$ & $7 \mathrm{de}$ & $0 \mathrm{f}$ & $50.0 \mathrm{a}$ & $3.5 \mathrm{efg}$ & $0.0 \mathrm{e}$ & $22 \mathrm{e}-\mathrm{h}$ \\
\hline Red Salad Bowl & $100 \mathrm{a}$ & $66 \mathrm{~b}$ & $29 \mathrm{~d}$ & $47.0 \mathrm{a}$ & $25.7 \mathrm{c}$ & $11.4 \mathrm{~d}$ & $3 \mathrm{jk}$ \\
\hline Red Flower & $100 \mathrm{a}$ & $2 \mathrm{e}$ & $0 \mathrm{f}$ & $50.0 \mathrm{a}$ & $1.0 \mathrm{fg}$ & $0.0 \mathrm{e}$ & $3 \mathrm{jk}$ \\
\hline Red Fox & $100 \mathrm{a}$ & $98 \mathrm{a}$ & $25 \mathrm{de}$ & $50.0 \mathrm{a}$ & $47.1 \mathrm{a}$ & $12.5 \mathrm{~d}$ & $12 \mathrm{~h}-\mathrm{k}$ \\
\hline Red Giant & $100 \mathrm{a}$ & $99 \mathrm{a}$ & $1 \mathrm{f}$ & $50.0 \mathrm{a}$ & $43.6 \mathrm{a}$ & $0.3 \mathrm{e}$ & $43 \mathrm{ab}$ \\
\hline Red Prize & $80 \mathrm{c}$ & $18 \mathrm{~d}$ & $2 \mathrm{f}$ & $40.0 \mathrm{~b}$ & $8.8 \mathrm{e}$ & $0.6 \mathrm{e}$ & $3 \mathrm{jk}$ \\
\hline Red Rage & $100 \mathrm{a}$ & $10 \mathrm{de}$ & $9 \mathrm{f}$ & $50.0 \mathrm{a}$ & $1.4 \mathrm{fg}$ & $1.0 \mathrm{e}$ & $15 \mathrm{~g}-\mathrm{j}$ \\
\hline Red Rebosa & $100 \mathrm{a}$ & $100 \mathrm{a}$ & $15 \mathrm{def}$ & $50.0 \mathrm{a}$ & $50.0 \mathrm{a}$ & $6.8 \mathrm{de}$ & $6 \mathrm{jk}$ \\
\hline Red Tide & $100 \mathrm{a}$ & $41 \mathrm{c}$ & $2 \mathrm{f}$ & $50.0 \mathrm{a}$ & $16.5 \mathrm{~d}$ & $1.0 \mathrm{e}$ & $1 \mathrm{k}$ \\
\hline Redina & $100 \mathrm{a}$ & $66 \mathrm{~b}$ & $2 \mathrm{f}$ & $50.0 \mathrm{a}$ & $33.0 \mathrm{~b}$ & $0.5 \mathrm{e}$ & $19 \mathrm{f}-\mathrm{i}$ \\
\hline Valdai & $100 \mathrm{a}$ & $100 \mathrm{a}$ & $75 \mathrm{~b}$ & $50.0 \mathrm{a}$ & $50.0 \mathrm{a}$ & $36.3 \mathrm{~b}$ & $25 \mathrm{~d}-\mathrm{g}$ \\
\hline
\end{tabular}

${ }^{\mathrm{z}}$ Means with the same letter in the same column are not significantly different at $P \leq 0.05$

Table 6. Effect of temperature on seed germination in wild species lettuce.

\begin{tabular}{|c|c|c|c|c|c|c|c|c|}
\hline \multirow[b]{2}{*}{ Genotype } & \multirow[b]{2}{*}{ Species } & \multicolumn{3}{|c|}{ Germination (\%) } & \multicolumn{3}{|c|}{ Germination rate } & \multirow{2}{*}{$\begin{array}{c}\text { Field } \\
\text { germination }(\%)\end{array}$} \\
\hline & & $24^{\circ} \mathrm{C}$ & $29^{\circ} \mathrm{C}$ & $34^{\circ} \mathrm{C}$ & $24^{\circ} \mathrm{C}$ & $29^{\circ} \mathrm{C}$ & $34^{\circ} \mathrm{C}$ & \\
\hline IVT 280 & VIR & $100 a^{z}$ & $100 \mathrm{a}$ & 76 abc & $50.0 \mathrm{a}$ & $48.7 \mathrm{ab}$ & $24.0 \mathrm{efg}$ & $1 \mathrm{ef}$ \\
\hline PI 187238 A & PRI & $100 \mathrm{a}$ & $100 \mathrm{a}$ & $91 \mathrm{ab}$ & $50.0 \mathrm{a}$ & $50.0 \mathrm{a}$ & $30.8 \mathrm{~b}-\mathrm{e}$ & $14 \mathrm{bc}$ \\
\hline PI 187238 D & PRI & $100 \mathrm{a}$ & $100 \mathrm{a}$ & $51 \mathrm{de}$ & $50.0 \mathrm{a}$ & $50.0 \mathrm{a}$ & $23.2 \mathrm{e}-\mathrm{h}$ & $13 \mathrm{bcd}$ \\
\hline PI 190906 & PRI & $100 \mathrm{a}$ & $100 \mathrm{a}$ & $69 \mathrm{~cd}$ & $48.5 \mathrm{ab}$ & $50.0 \mathrm{a}$ & $19.8 \mathrm{gh}$ & $15 \mathrm{bc}$ \\
\hline PI 202349 C & PRI & $100 \mathrm{a}$ & $100 \mathrm{a}$ & $7 \mathrm{~g}$ & $50.0 \mathrm{a}$ & $50.0 \mathrm{a}$ & $2.3 \mathrm{i}$ & $19 \mathrm{~b}$ \\
\hline PI 204753 & PRI & $95 \mathrm{a}-\mathrm{d}$ & $8 \mathrm{~h}$ & $0 \mathrm{~g}$ & $46.8 \mathrm{a}-\mathrm{d}$ & $2.1 \mathrm{i}$ & $0.0 \mathrm{i}$ & 5 ef \\
\hline PI 271938 & VIR & $100 \mathrm{a}$ & $100 \mathrm{a}$ & $78 \mathrm{abc}$ & $49.3 \mathrm{ab}$ & $50.0 \mathrm{a}$ & $22.0 \mathrm{fgh}$ & $7 \mathrm{c}-\mathrm{f}$ \\
\hline PI $274378 \mathrm{~A}$ & VIR & $94 \mathrm{bcd}$ & $86 \mathrm{~d}$ & 41 ef & $42.8 \mathrm{e}$ & $34.9 \mathrm{~d}$ & $5.7 \mathrm{i}$ & $2 \mathrm{ef}$ \\
\hline PI 274378 D & VIR & $100 \mathrm{a}$ & $98 \mathrm{abc}$ & $86 \mathrm{abc}$ & $43.8 \mathrm{cde}$ & $45.5 \mathrm{bc}$ & $31.0 \mathrm{~b}-\mathrm{e}$ & 8 cde \\
\hline PI 274901 & VIR & $96 \mathrm{a}-\mathrm{d}$ & $100 \mathrm{a}$ & $75 \mathrm{bc}$ & $48.0 \mathrm{ab}$ & $50.0 \mathrm{a}$ & $36.1 \mathrm{abc}$ & $17 \mathrm{~b}$ \\
\hline PI 281877 & SER & $100 \mathrm{a}$ & $6 \mathrm{~h}$ & $0 \mathrm{~g}$ & $50.0 \mathrm{a}$ & $3.0 \mathrm{i}$ & $0.0 \mathrm{i}$ & $4 \mathrm{ef}$ \\
\hline PI 289063 A & PRI & $100 \mathrm{a}$ & $100 \mathrm{a}$ & $79 \mathrm{abc}$ & $50.0 \mathrm{a}$ & $50.0 \mathrm{a}$ & $38.9 \mathrm{ab}$ & $33 \mathrm{a}$ \\
\hline PI $289063 \mathrm{C}$ & PRI & $100 \mathrm{a}$ & $100 \mathrm{a}$ & $82 \mathrm{abc}$ & $50.0 \mathrm{a}$ & $50.0 \mathrm{a}$ & $25.1 \mathrm{efg}$ & $13 \mathrm{bc}$ \\
\hline PI 490999 & SAL & $98 \mathrm{abc}$ & $100 \mathrm{a}$ & $52 \mathrm{de}$ & $48.8 \mathrm{ab}$ & $50.0 \mathrm{a}$ & $24.3 \mathrm{efg}$ & $0 \mathrm{f}$ \\
\hline PI 491000 & SAL & $93 \mathrm{~cd}$ & $35 \mathrm{~g}$ & $31 \mathrm{f}$ & $46.0 \mathrm{~b}-\mathrm{e}$ & $15.8 \mathrm{~g}$ & $15.1 \mathrm{~h}$ & $1 \mathrm{ef}$ \\
\hline PI 491089 & SER & $100 \mathrm{a}$ & $53 \mathrm{f}$ & $1 \mathrm{~g}$ & $48.5 \mathrm{ab}$ & $5.4 \mathrm{i}$ & $0.1 \mathrm{i}$ & $0 \mathrm{f}$ \\
\hline PI 491112 & SER & $100 \mathrm{a}$ & $100 \mathrm{a}$ & $94 \mathrm{a}$ & $48.0 \mathrm{ab}$ & $50.0 \mathrm{a}$ & $37.9 \mathrm{ab}$ & $1 \mathrm{ef}$ \\
\hline PI 491119 & SER & $100 \mathrm{a}$ & $100 \mathrm{a}$ & $75 \mathrm{bc}$ & $50.0 \mathrm{a}$ & $50.0 \mathrm{a}$ & $29.7 \mathrm{c}-\mathrm{f}$ & 3 ef \\
\hline PI 491134 & SER & $100 \mathrm{a}$ & $100 \mathrm{a}$ & 74 bc & $50.0 \mathrm{a}$ & $50.0 \mathrm{a}$ & $27.5 \mathrm{~d}-\mathrm{g}$ & $5 \mathrm{def}$ \\
\hline PI 491147 & SER & $100 \mathrm{a}$ & $87 \mathrm{~cd}$ & $86 \mathrm{abc}$ & $50.0 \mathrm{a}$ & $41.8 \mathrm{c}$ & $33.5 \mathrm{bcd}$ & 5 ef \\
\hline PI 491159 & SAL & $91 \mathrm{~d}$ & 88 bcd & $79 a b c$ & $43.5 \mathrm{de}$ & $21.5 \mathrm{f}$ & $23.8 \mathrm{efg}$ & $5 \mathrm{ef}$ \\
\hline PI 491204 & SAL & $100 \mathrm{a}$ & $54 \mathrm{f}$ & $53 \mathrm{de}$ & $50.0 \mathrm{a}$ & $10.1 \mathrm{~h}$ & $26.0 \mathrm{~d}-\mathrm{g}$ & $2 \mathrm{ef}$ \\
\hline PI 491205 & SAL & $99 \mathrm{ab}$ & $71 \mathrm{e}$ & $51 \mathrm{de}$ & $48.5 \mathrm{ab}$ & $33.2 \mathrm{~d}$ & $23.7 \mathrm{efg}$ & $1 \mathrm{ef}$ \\
\hline PI 491206 & SAL & $100 \mathrm{a}$ & $73 \mathrm{e}$ & 49 ef & $49.0 \mathrm{ab}$ & $28.6 \mathrm{e}$ & $24.0 \mathrm{efg}$ & $0 \mathrm{f}$ \\
\hline PI 491207 & SAL & $98 \mathrm{abc}$ & $100 \mathrm{a}$ & 37 ef & $47.3 \mathrm{abc}$ & $50.0 \mathrm{a}$ & $15.3 \mathrm{~h}$ & $0 \mathrm{f}$ \\
\hline UC96US23 & SER & $100 \mathrm{a}$ & $99 \mathrm{ab}$ & $94 \mathrm{a}$ & $48 \mathrm{ab}$ & $44 \mathrm{c}$ & $43 \mathrm{a}$ & nd \\
\hline
\end{tabular}

${ }^{\mathrm{z}}$ Means with the same letter in the same column are not significantly different at $P \leq 0.05$.

$\mathrm{VIR}=$ L. virosa $; \mathrm{SAL}=$ L. saligna $; \mathrm{PRI}=$ primitive; $\mathrm{SER}=$ L. serriola

lettuce by temperature may involve plant hormones such as ABA and GA. High temperature stimulated ABA synthesis and inhibited GA synthesis in imbibed Arabidopsis seeds (Toh et al., 2008). The increase in ABA levels in seeds of the Salinas cultivar that exhibited thermoinhibition indicated that ABA plays a key role in thermoinhibition of lettuce seeds (Argyris et al., 2008a; Nambara et al., 2010). 'Salinas' also exhibited thermoinhibition at 29 to $34{ }^{\circ} \mathrm{C}$ as observed in this study. The observed increase in thermotolerance in UC96US23 was associated with a decrease in ABA biosynthesis at high temperature (Argyris et al., 2011). Seed covering may also be involved in regulation of seed germination by imposing a restriction on seed germination at high temperature (Ikuma and Thimann, 1963; Speer, 1974; Sung et al., 1998). The stimulation of activities of hydrolytic enzymes is required to promote seed germination and embryo growth (Khan, 1994; Nascimento et al., 2000)

The results of this study indicated that seeds of cultivars and germplasm of various lettuce types (crisphead, romaine, butterhead,
Table 7. Correlation coefficients of percentage seed germination $\left(24,29,34{ }^{\circ} \mathrm{C}\right.$ and field) among lettuce genotypes.

\begin{tabular}{|c|c|c|c|c|}
\hline $\begin{array}{l}\text { Lettuce } \\
\text { type }\end{array}$ & Germination & $24{ }^{\circ} \mathrm{C}$ & $29^{\circ} \mathrm{C}$ & $34^{\circ} \mathrm{C}$ \\
\hline \multirow[t]{3}{*}{ Butterhead } & $29^{\circ} \mathrm{C}$ & $0.39 *$ & & \\
\hline & $34{ }^{\circ} \mathrm{C}$ & 0.30 & $0.65 * *$ & \\
\hline & Field & 0.37 & $0.57 * *$ & $0.41 *$ \\
\hline \multirow[t]{3}{*}{ Crisphead } & $29^{\circ} \mathrm{C}$ & 0.28 & & \\
\hline & $34{ }^{\circ} \mathrm{C}$ & -0.10 & $0.42 *$ & \\
\hline & Field & 0.28 & 0.38 & 0.33 \\
\hline \multirow[t]{3}{*}{ Romaine } & $29^{\circ} \mathrm{C}$ & -0.14 & & \\
\hline & $34^{\circ} \mathrm{C}$ & -0.18 & $0.75^{* *}$ & \\
\hline & Field & -0.20 & $0.73 * *$ & $0.68 * *$ \\
\hline \multirow[t]{3}{*}{ Green leaf } & $29^{\circ} \mathrm{C}$ & 0.36 & & \\
\hline & $34{ }^{\circ} \mathrm{C}$ & 0.24 & $0.48 *$ & \\
\hline & Field & 0.30 & 0.20 & $0.57 * *$ \\
\hline \multirow[t]{3}{*}{ Red leaf } & $29^{\circ} \mathrm{C}$ & 0.40 & & \\
\hline & $34{ }^{\circ} \mathrm{C}$ & 0.18 & $0.47 *$ & \\
\hline & Field & 0.30 & $0.65 * *$ & $0.52 * *$ \\
\hline \multirow{3}{*}{$\begin{array}{l}\text { Wild } \\
\text { species }\end{array}$} & $29^{\circ} \mathrm{C}$ & 0.28 & & \\
\hline & $34{ }^{\circ} \mathrm{C}$ & 0.18 & $0.72 * *$ & \\
\hline & Field & 0.20 & 0.35 & 0.23 \\
\hline \multirow{3}{*}{$\begin{array}{l}\text { All lettuce } \\
\text { types }\end{array}$} & $29^{\circ} \mathrm{C}$ & $0.28 * *$ & & \\
\hline & $34^{\circ} \mathrm{C}$ & 0.12 & $0.56^{* *}$ & \\
\hline & Field & $0.27 * *$ & $0.46 * *$ & $0.34 * *$ \\
\hline
\end{tabular}

$*$,**Significant correlations at $5 \%$ and $1 \%$ levels of probability, respectively.

green and red leaf, and primitive and wild species) differed greatly in their ability to germinate at 29 and $34^{\circ} \mathrm{C}$. However, at $24^{\circ} \mathrm{C}$, seeds of most lettuce genotypes germinated rapidly and uniformly. Some lettuce cultivars and germplasm exhibited thermoinhibition at $29^{\circ} \mathrm{C}$, whereas others exhibited thermotolerance at high temperature $\left(34^{\circ} \mathrm{C}\right)$. The maximum temperatures for thermoinhibition may depend on lettuce genotype. Despite the variations in field germination, seed germination in the field positively correlated with seed germination at 29 and $34^{\circ} \mathrm{C}$. This evaluation of seed germination at 29 and $34{ }^{\circ} \mathrm{C}$ helped in identifying lettuce cultivars and germplasm that tolerate high-temperature stress. Selecting lettuce cultivars with good germination at high temperatures is essential to ensure uniform stand establishment and subsequent uniform maturity at harvest.

Most U.S. lettuce production is carried out in the central coast of California with transit to the San Joaquin Valley for a short period in spring and fall and a switch to southern California and Arizona for winter crops. Land costs in the coastal production areas are usually several times higher than in the inland regions. However, production seasons in these low land cost areas are limited by heat stress and thermoinhibition. Seed priming is commonly used to prevent thermodormancy and ensure uniform emergence, even in coastal production areas. Thermoinsensitive lettuce varieties could help expand the production seasons in warm and low land 
cost areas nationwide and reduce the need for seed priming, lowering the production costs. As the costs of land, labor, fuel, fertilizer, pesticides, seeds, packing material, cooling, transportation, and overhead including food safety continue to rise, it is essential to reduce production costs of leafy vegetables to benefit producers as well as consumers.

The results from this study may help growers choose lettuce varieties to be grown in a warm environment. These data may also help lettuce breeders to improve the crop for resistance to heat stress. A breeding program usually starts from germplasm screening to find the source of beneficial traits. Because the development of a new lettuce cultivar may take up to 10 years, there is an urgent need to breed thermodormancy-resistant cultivars for adaptation to global warming and climate changes.

\section{Literature Cited}

Argyris, J., P. Dahal, E. Hayashi, D.W. Still, and K.J. Bradford. 2008a. Genetic variation for lettuce seed thermoinhibition is associated with temperature-sensitive expression of abscisic acid, gibberellin, and ethylene biosynthesis, metabolism, and response genes. Plant Physiol. 148:926-947.

Argyris, J., P. Dahal, M.J. Truco, O. Ochoa, D.W. Still, R.W. Michelmore, and K.J. Bradford. 2008b. Genetic analysis of lettuce seed thermoinhibition. Acta Hort. 782:23-33.

Argyris, J., M.J. Truco, O. Ochoa, L. McHale, P. Dahal, A.V. Deynze, R.W. Michelmore, and K.J. Bradford. 2011. A gene encoding an abscisic acid biosynthesis enzyme ( $L S N C E D 4)$ collocates with the high temperature germination locus Htg6.1 in lettuce (Lactuca sp.). Theor. Appl. Genet. 122:95-108.

Bradford, K.J. 1986. Manipulation of seed water relations via osmotic priming to improve germination under stress conditions. HortScience 21:1105-1112.

Bradford, K.J. and O.A. Somasco. 1994. Water relations of lettuce seed thermoinhibition. I. Priming and endosperm effects on base water potential. Seed Sci. Res. 4:1-10.

Cantliffe, D.J., K.D. Shuler, and A.C. Guedes. 1981. Overcoming seed thermodormancy in a heat-sensitive romaine lettuce by seed priming. HortScience 16:196-198.

Coons, J.M., R.O. Kuehl, and N.R. Simons. 1990. Tolerance of ten lettuce cultivars to high temperature combined with $\mathrm{NaCl}$ during germination. J. Amer. Soc. Hort. Sci. 115:1004 1007.

Deng, Z. and S. Song. 2012. Sodium nitroprusside, ferricyanide, nitrite and nitrate decrease the thermo-dormancy of lettuce seed germination in a nitric oxide-dependent manner in light. $\mathrm{S}$. Afr. J. Bot. 78:139-146.

Dutta, S. and K.J. Bradford. 1994. Water relations of lettuce seed thermoinhibition. II. Ethylene and endosperm effects on base water potential. Seed Sci. Res. 4:11-18.

Gonai, T., S. Kawahara, M. Tougou, S. Satoh, T. Hashiba, N. Hirai, H. Kawaide, Y. Kamiya, and T. Yoshioka. 2004. Abscisic acid in the thermoinhibition of lettuce seed germination and enhancement of its catabolism by gibberellin. J. Expt. Bot. 55:111-118.

Gray, D. 1975. Effect of temperature on the germination and emergence of lettuce (Lactica sativa) varieties. J. Hort. Sci. 50:349-361.

Huang, X. and A.A. Khan. 1992. Alleviation of thermoinhibition in preconditioned lettuce seeds involves ethylene, not polyamine biosynthesis. J. Amer. Soc. Hort. Sci. 117:841-845.

Ikuma, H. and K.V. Thimann. 1963. The role of seed-coats in germination of photosensitive lettuce seeds. Plant Cell Physiol. 4:169-185.

Jenni, S. 2005. Rib discoloration: A physiological disorder induced by heat stress in crisphead lettuce. HortScience 40:2031-2035.

Jenni, S. and W. Yan. 2009. Genotype by environment interactions of heat stress disorder resistance in crisphead lettuce. Plant Breed. 128: 374-380.

Jett, L.W., G.E. Welbaum, and R.D. Morse. 1996. Effect of matric and osmatic priming treatments on broccoli seed germination. J. Amer. Soc. Hort. Sci. 121:423-429.

Karl, T.R. and K.E. Trenberth. 2003. Modern global climate change. Science 302:1719-1723.

Khan, A. 1994. Induction of dormancy in nondormant seeds. J. Amer. Soc. Hort. Sci. 119: 408-413.

Khan, A.A. and J. Prusinski. 1989. Kinetin enhanced 1-aminocyclopropane-1-carboxylic acid utilization during alleviation of high temperature stress in lettuce seeds. Plant Physiol. 91:733-737.

Kozarewa, I., D.J. Cantliffe, R.T. Nagata, and P.J. Stoffella. 2006. High maturation temperature of lettuce seeds during development increased ethylene production and germination at elevated temperatures. J. Amer. Soc. Hort. Sci. 131:564-570.

Kucera, B., M.A. Cohn, and G. Leubner-Metzger. 2005. Plant hormone interactions during seed dormancy release and germination. Seed Sci. Res. 15:281-307.
Meguire, J.D. 1962. Speed of germination-Aid in selection and evaluation for seedling emergence and vigor. Crop Sci. 2:176-177.

Nambara, E., M. Okamoto, K. Tatematsu, R. Yano, M. Seo, and Y. Kamiya. 2010. Abscisic acid and the control of seed dormancy and germination. Seed Sci. Res. 20:55-67.

Nascimento, W.M., D.J. Cantliffe, and D.J. Huber. 2000 . Endo- $\beta$-mannanase activity during lettuce seed germination at high temperature conditions. Acta Hort. 517:107-112.

Negm, F.B., O.E. Smith, and J. Kumamoto. 1972. Interaction of carbon dioxide and ethylene in overcoming thermodormancy of lettuce seeds. Plant Physiol. 49:869-872.

Samfield, D.M., J.M. Zajicek, and B.G. Cobb. 1991. Rate and uniformity of herbaceous perennial seed germination and emergence as affected by priming. J. Amer. Soc. Hort. Sci. 116:10-13.

Speer, H.L. 1974. Some aspects of the function of the endosperm during the germination of lettuce seeds. Can. J. Bot. 52:1117-1121.

Sung, Y., D.J. Cantliffe, and R.T. Nagata. 1998. Seed developmental temperature regulation of thermotolerance in lettuce. J. Amer. Soc. Hort. Sci. 123:700-705.

Sung, Y., D.J. Cantliffe, R.T. Nagata, and W.M. Nascimento. 2008. Structural changes in lettuce seed during germination at high temperature altered by genotype, seed maturation temperature, and seed priming. J. Amer. Soc. Hort. Sci. 133:300-311.

Thompson, P.A., S.A. Cox, and R.H. Sanderson. 1979. Characterization of the germination responses to temperature of lettuce (Lactuca sativa L.) achenes. Ann. Bot. (Lond.) 43:319-334.

Toh, S., A. Imamura, A. Watanabe, K. Nakabayashi, M. Okamoto, Y. Jikumaru, A. Hanada, Y. Aso, K. Ishiyama, S. Iuchi, M. Kobayashi, S. Yamaguchi, Y. Kamiya, E. Nambara, and N. Kawakami. 2008. High temperature-induced abscisic acid biosynthesis and its role in the inhibition of gibberellin action in Arabidopsis seeds. Plant Physiol. 146:1368-1385.

Valdes, V.M., K.J. Bradford, and K.S. Mayberry. 1985. Alleviation of thermodormancy in coated lettuce seeds by seed priming. HortScience 20:1112-1114.

Vidaver, W. and A.I. Hsiao. 1975. Actions of gibberellic acid and phytochrome on the germination of Grand Rapids lettuce seeds. Plant Physiol. 53:266-268.

Wurr, D.C.E., J.R. Fellows, and K. Phelps. 1996. Investigating trends in vegetable crop response to increasing temperature associated with climate change. Sci. Hort. 66:255-263. 